\title{
An Efficient Power Management for Wireless Sensor Systems
}

\author{
Jina Hong and Namgi Kim ${ }^{1}$ \\ Department of Computer Science, Kyonggi University \\ \{jin-a,ngkim\}@kgu.ac.kr
}

\begin{abstract}
Devices existing within a wireless sensor system are small in size and their battery capacities are very limited. In addition, diverse channel environments may be configured for them. To overcome various constraints and efficiently utilize the energy of sensor nodes, transmission power control (TPC) technology may be employed. This paper describes our attempt to resolve problems with existing TPC algorithms, reduce the number of unnecessary changes in transmission power level (TPL) using the slope value of linear regressed equation, and present an algorithm for deriving TPL in a fast and appropriate manner. Through an experiment, in this study, we not only derive proper weight value of the proposed algorithm but also compare its performance with that of existing algorithms by analyzing the numbers of control packets, drop packets, and energy consumption.
\end{abstract}

Keywords: transmission power control, wireless body sensor system, transmission power level, energy consumption

\section{Introduction}

Recently, as sensor devices have become small in size and the development of wireless communication technologies is accelerating, wireless sensor systems (WSSs) are attracting attention [1]. Among them is a new type of system called wireless body sensor system (WBSS) in which sensor devices are dispatched inside or outside with the body area as the center and inter-device communication is possible. In particular, WBSS standardization centered on the IEEE 802.15.6 BAN Task Group [2] is progressing actively owing to improved quality of life, interest in welfare, and the onset of aging societies. Besides, research intending to combine a WBSS with medical technologies or IT in the industrial, academic, and research worlds is being pursued keenly.

Unlike existing networks that focus on finding data delivery paths, a WBSS has additional constraints that need to be analyzed [3]. First, WBSSs are installed on the human body; therefore, the devices are small, and their space saving capacity is small. Second, humans install them in and on the body; therefore, battery power onboard the devices is low and swapping batteries is difficult. Third, the received signal strength indication (RSSI) differs with physical channel condition elements - users' movements, location of sensor nodes, and their place of utilization. Therefore, research on efficient use of device energy considering channel conditions in WBSSs is important.

Low-power media access control (MAC) technology [4] and transmission power control (TPC) method can be used to operate sensor nodes in an energy-efficient manner. The low power MAC technology saves energy by removing major causes of energy waste-collision, overhearing, control packet overhead, and idle listening - in wireless data communications [5]. The TPC method saves energy by controlling transmission power when a sensor node

\footnotetext{
${ }^{1}$ Corresponding author: Namgi Kim

This paper is a revised and expanded version of a paper entitled "An Empirical Study on Channel Characteristics of Wireless Body Sensor Networks"presented at ASEA 2014, Haikou, China, and December 21th 2014.
} 
sends a data packet to a sink node. This work aims to save energy at the sensor node using the TPC method.

\section{Related Work}

In the WSS area, considerable research has been conducted on increasing energy efficiency by controlling transmission power [6]. In particular, representative algorithms for implementing TPC include linear [7], binary [8], and dynamic [9] algorithms. These algorithms are used to find the next transmission power line (TPL) when RSSI goes beyond the target RSSI margin range. Figure 1 shows changes in the TPL and RSSI values in the case that sink nodes use the TPC algorithm when RSSI rises beyond the target RSSI margin in a body sensor system environment. In this figure, the $\mathrm{x}$-axis, $\mathrm{y}$-axis on the left side, and y-axis on the right side of the graph denote time, RSSI, and TPL, respectively.

A linear algorithm is the simplest exploration algorithm used to find a certain TPL. It controls by reading the RSSI value and increasing or decreasing the TPL by a level as suitable. This operation method is very efficient because the TPL is changed by one level when the channel is unstable. However, when the channel is stable, the number of changes in TPL is too large to reach an optimal TPL. Therefore, it is difficult to reflect the most recent channel condition, and at every such moment, a control packet is sent, which results in energy consumption due to control packet overuse. As shown in Figure 1(a), a linear algorithm would need multi-level TPL changes for finding an optimal TPL when the channel environment changes. In the presence of a considerable difference between the existing channel condition and the revised channel condition, the outcome is unfavorable.

In contrast, the binary algorithm operates by exponentially increasing or decreasing TPL. If the current RSSI drops below the lower bound of the target RSSI margin, the next TPL level is selected to be in the middle of the current value and the maximal value. On the contrary, if the current RSSI rises above the higher bound of the target RSSI margin, the next TPL level is selected to be between in the middle of the current value and the minimal value. As can be seen in Figure 1(b), even though the TPL level changes, an optimal TPL is not found. Moreover, in many cases, the TPL goes beyond the target RSSI margin, and therefore, an oscillation with up and down vibration appears. In this case, in contrast with the linear algorithm, an appropriate TPL is found very swiftly when the channel is stable, thus allowing for greater energy saving than that possible with other algorithms. Nonetheless, the binary algorithm unnecessarily controls TPL when the channel is unstable and sets an inappropriate level for the next TPL. In this case, control packets are sent excessively frequently, leading to excessive energy consumption not only for control packet transmission at the sink node but also for control packet reception at the sensor node. Moreover, inappropriate TPL establishment increases energy consumed in data packet movement. 


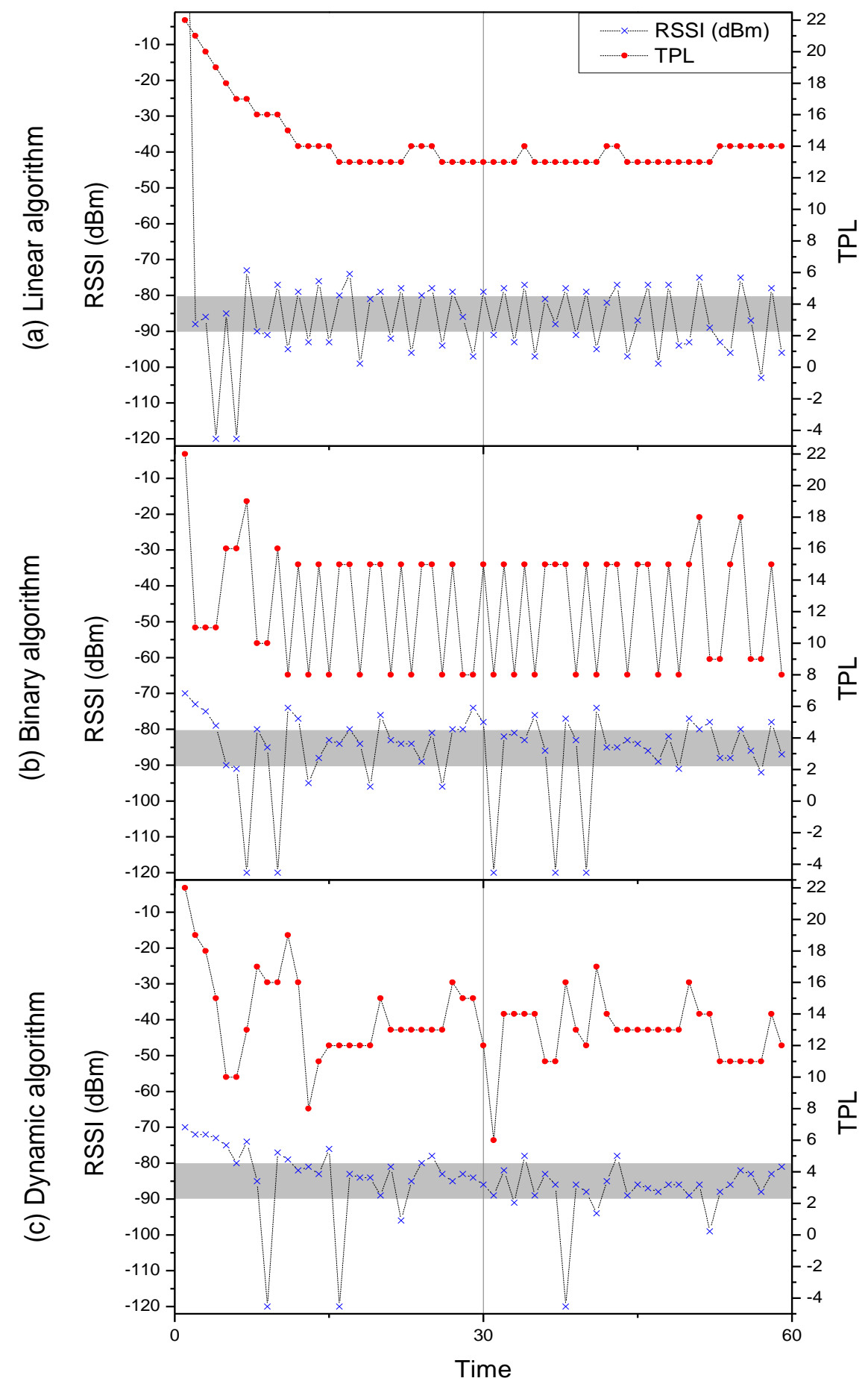

Figure 1. Changes in Transmission Power Level and RSSI when Using an Existing Algorithm

The dynamic algorithm derives a linear equation to determine the next TPL. First, in order to create an equation, two RSSI values are needed; a new linear equation is derived using the two values. Then, an additional linear equation is derived using the next RSSI value. Therefore, the dynamic algorithm covers the disadvantages of the linear and binary algorithms to an extent, but similar to the binary algorithm, execution of this algorithm turns abnormal in a body sensor system environment in which the channel environment changes frequently. This results in many cases of inefficient algorithm operation, as shown in Figure 1(c). To overcome the aforementioned disadvantages of the representative algorithms, in this 
study, we used a moving average technique to determine an optimal TPL by efficiently collecting RSSI values of data packets and generating a linear equation that considers WBSS characteristics by using the collected RSSI values.

\section{Proposed Algorithm}

Figure 2 shows the flowchart of the algorithm proposed in this paper. This algorithm operates as follows. When the sink node receives the relevant data packet, it immediately measures the current RSSI. The calculated average RSSI is used to judge whether it is within the target RSSI margin range. A moving average is used to calculate the average RSSI in the proposed algorithm. This is because when judging whether the average RSSI is within the target RSSI margin by using the current RSSI, RSSI deviation cannot be overcome, and when the channel is unstable, an excessive number of control packets are used. Therefore, average RSSI was calculated using (1) and 0.125 was used as the weight.

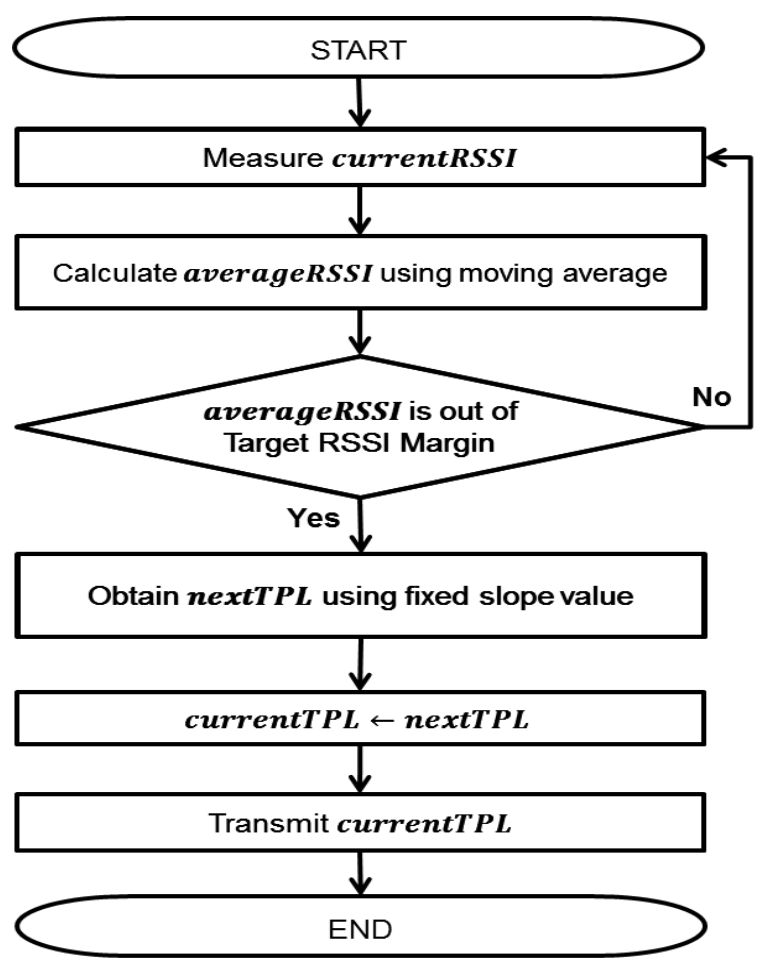

Figure 2. Flowchart of Proposed Algorithm

After calculating the average RSSI, we determine whether this value is larger than the upper RSSI margin or smaller than the lower RSSI margin. If the calculated value is within the target RSSI margin, a data packet is sent using the same TPL as that for the previous packet; if it goes beyond the target RSSI margin, a new linear equation with the same slope but a different y intercept is derived. Using this linear equation, a newt TPL that can approximate the target RSSI point is calculated, and the current TPL is modified. 


\section{Experiment Environment}

\subsection{Experiment System Architecture}

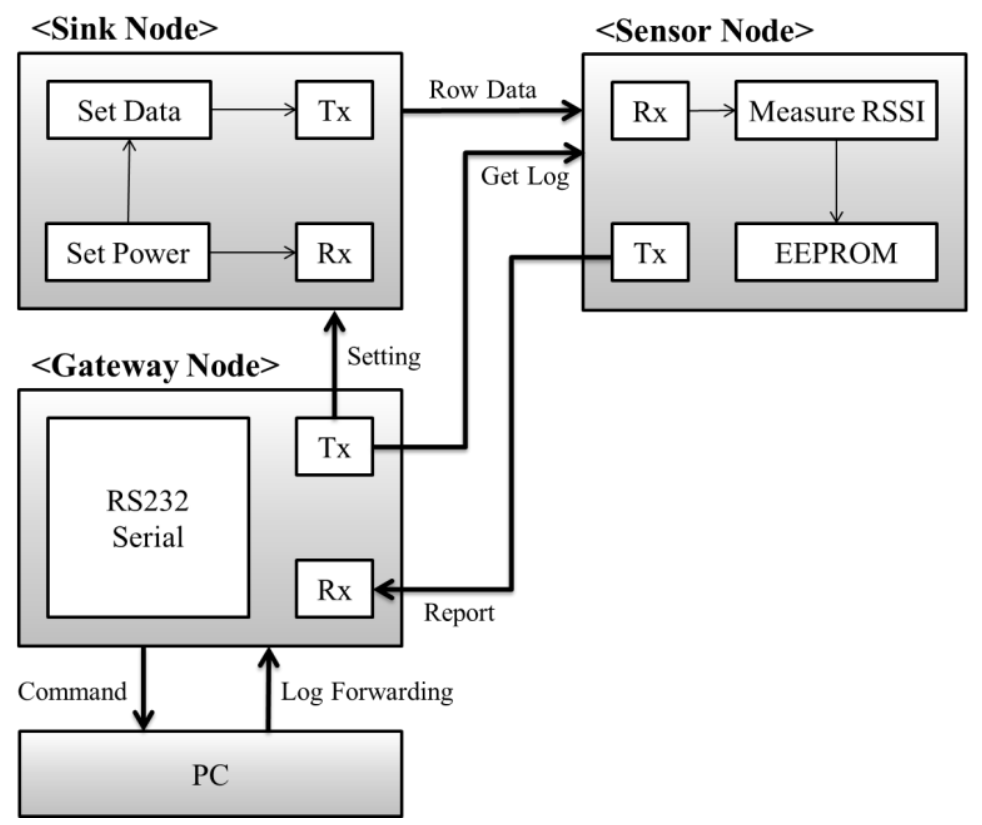

Figure 3. System Architecture of Experimental Setup

Figure 3 shows the structure of a system composed of a personal computer, gateway node, sink node, and sensor node. The execution of this system takes place in the following order using the sensor node that is explained below. The user sends a command to the gateway node through an RS232 serial port on the computer. The gateway node sends the command to the sink node. Then, the sink node sets the current power level, timer, and log data based on the received command. According to the set timer, the sink node sends data to the sensor node, and the sensor nodes measures the RSSI value of the received data and stores log data in electrically erasable programmable read-only memory (EEPROM). In case the gate node requests for a log record from the sensor node through the Get Log command, the sensor node places the measured value stored in the EEPROM in Report Message and sends it to the gateway node. The gateway node forwards the log.

\subsection{Experimental Environment Setting}

This paper used an actual sensor mote to create a channel environment for the body sensor system. The device used here, shown in Figure 4, is the Cricket Mote produced by Crossbow Technology [10]. The Cricket Mote operates with two $1.5 \mathrm{~V}$ batteries in the $433 \mathrm{MHz}$ frequency band. The Cricket Mote has a CC1000 radio module-based [11] TPL consisting of 23 levels. Because changes in the radio chip result in changes in device characteristics, transmission current, power consumption, and speed may change [12]. 


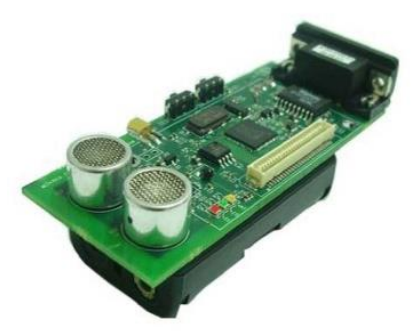

Figure 4. Sensor Device

Table 1. Sensor Characteristics and Experimental Environment Setting

\begin{tabular}{cc|cc}
\hline Sensor properties & Values & Experimental setups & Values \\
\hline \hline Radio chips & CC1000 & Sensor placement & Stomach, Back \\
Supply voltage & $2.5 \mathrm{~V}$ & Sink placement & Chest \\
Radio frequency & $433 \mathrm{MHz}$ & Body movements & Standing, Running \\
Transmission bit & $19.2 \mathrm{kbps}$ & Target RSSI point & $-85 \mathrm{dBm}$ \\
$\begin{array}{c}\text { rate } \\
\text { Output power } \\
\text { Transmission } \\
\text { current }\end{array}$ & -20 to $-10 \mathrm{dBm}$ & Target RSSI margin & -90 to $-80 \mathrm{dBm}$ \\
Reception current & 6.9 to $26.7 \mathrm{~mA}$ & Sensing period & $1 \mathrm{~s}$ \\
\hline
\end{tabular}
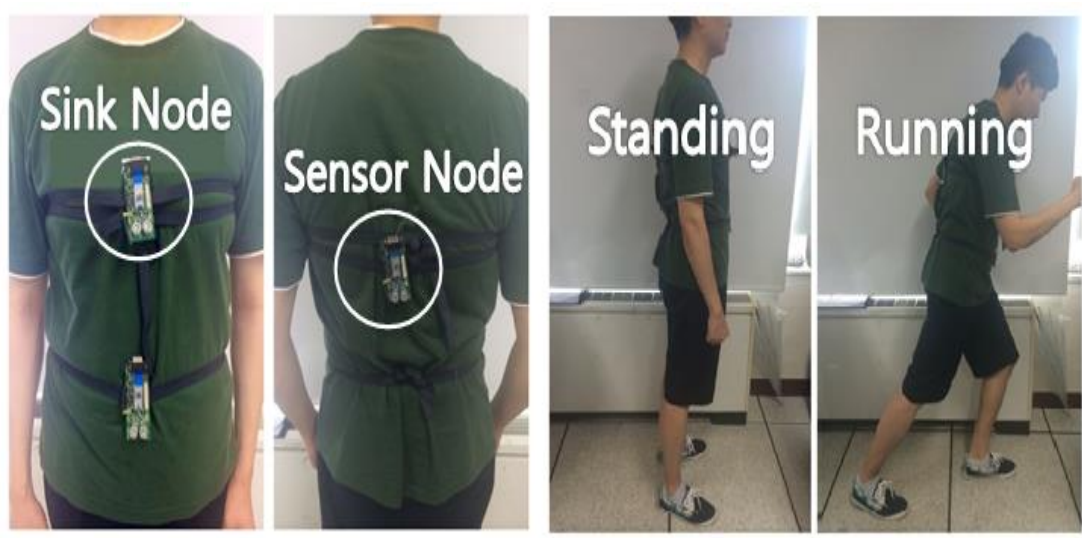

Figure 5. Node Locations and Subject's Movements

Table 1 summarizes the system and the environmental variables. In the body sensor system environment, diverse channel conditions may be formed depending on the locations of the sensor and sink nodes, subject's movements, and place of experiment. In this study, the sink node was located on the chest and the sensor nodes were located on the abdomen and the back, as shown in Figure 6. The experiment was conducted under both conditions: line of sight (LOS) and non-line of sight (Non-LOS). As shown in Figure 5, the subject's movement included standing and jumping, and the experiment was conducted in a laboratory (indoor) and a playground (outdoor). Because the target RSSI point was $-85 \mathrm{dBm}$ and the target RSSI size was $\pm 5 \mathrm{dBm}$, the target RSSI margin ranged from -90 to $-80 \mathrm{dBm}$. This value range is appropriate for this experiment's body sensor system. A data packet was collected with a cycle of $1 \mathrm{~s}$, and the experiment duration was 720 seconds. 


\subsection{Experimental Body Movement Scenario}

Figure 6 displays the body movement scenario established considering the external factors explained earlier. This scenario combines all conditions of the indoor and outdoor experimental locations and various subject movements such as standing and jumping. The total number of states is 12. Each state represents experimental location/subject movement. Under the indoor/standing condition at the beginning, 60 data packets were sent for each state. The total cycle involved one repetition of all states.

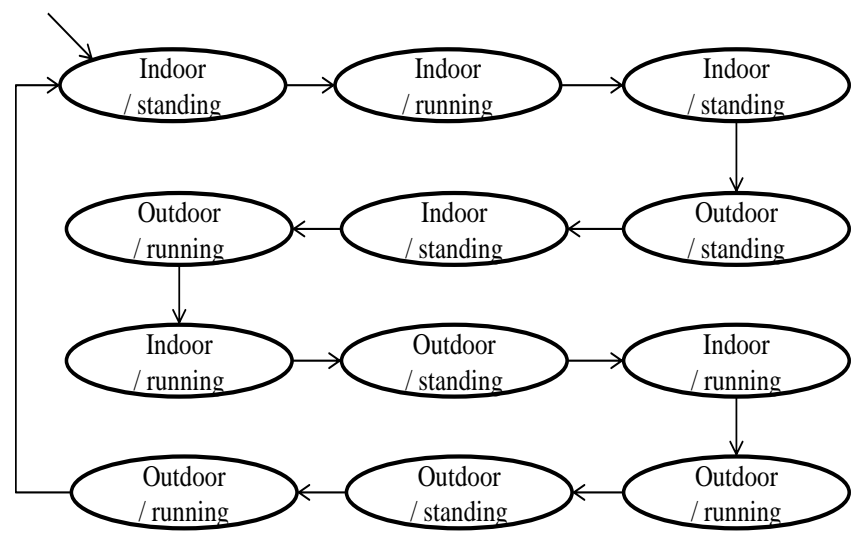

Figure 6. Body Movement Scenario

\section{Experiment Results}

\subsection{Energy Consumption According to Weight}

In order to examine the effect of setting the weight value on energy consumption in calculating average RSSI, diverse weight values were used in the experiment. Figure 7 shows the number of control packets and average TPL according to various weight settings. The $\mathrm{x}$ axis represents the weight value, y-axis on the left side represents the number of control packets, and y-axis on the right side represents the average TPL value. The lower the weight value, the greater is the weight of average RSSI and the smaller is the weight of current RSSI, as can be inferred from Table 2. Therefore, in the case of 0.03125 , the number of control packets is the smallest because response to changes in the channel is slow, which reduces the number of control packets. With fewer control packets, the energy consumed for sending and receiving control packet is reduced, thus resulting in a reduction in overall energy consumption.

However, the average TPL is the lowest at 11.62 when the weight is 0.125 rather than 0.03125 . When the number of control packets is small, even though the data packet is sent with inappropriate TPL, changes in channel are perceived slowly, resulting in slow changes in TPL. As a result, the energy consumed to send and receive data packet increases, which may affect total energy consumption.

Table 2. Weight Value

\begin{tabular}{c|cc}
\hline Weight & Average RSSI & Current RSSI \\
\hline \hline 0.03125 & 0.9688 & 0.03125 \\
0.0625 & 0.9375 & 0.0625 \\
0.125 & 0.875 & 0.125 \\
0.25 & 0.75 & 0.25 \\
0.5 & 0.5 & 0.5 \\
\hline
\end{tabular}




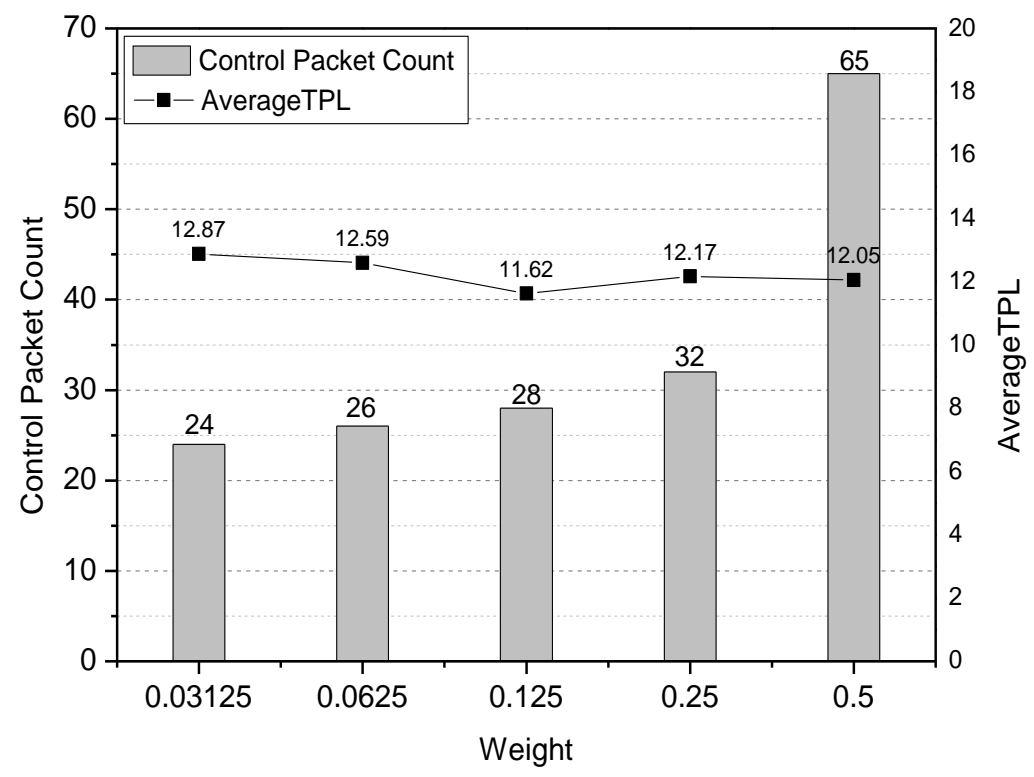

Figure 7. Number of Control Packets and Average TPL According to Weight

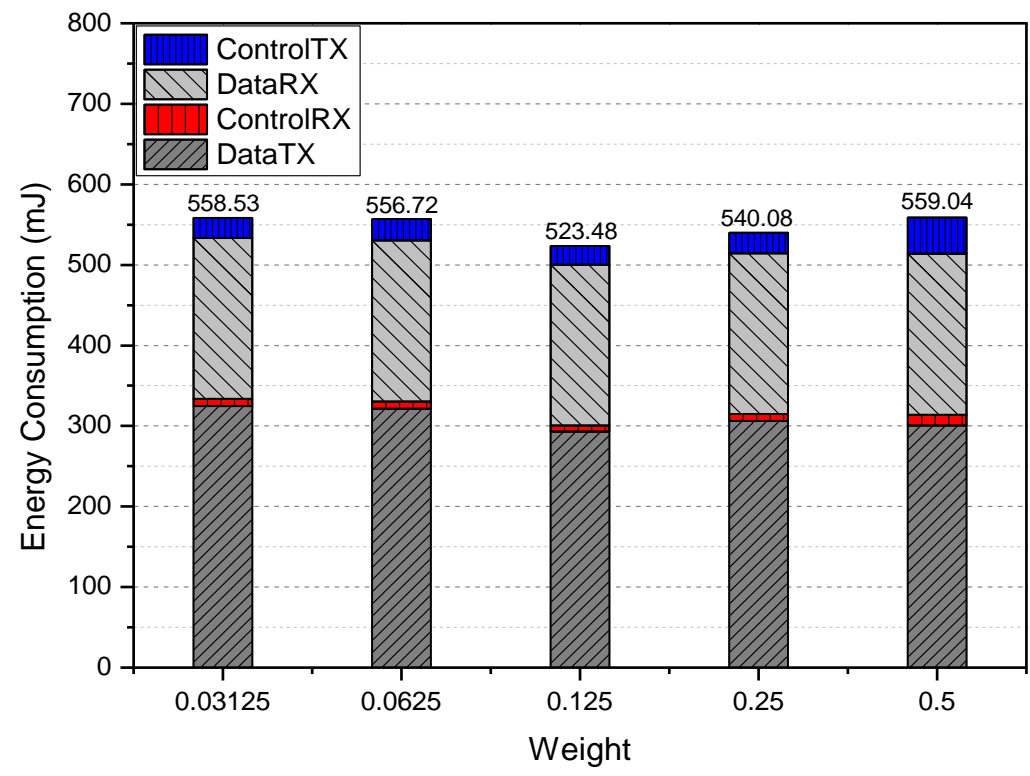

Figure 8. Number of Control Packets and Average TPL According to Weight

Figure 8 shows the total energy consumption according to the weight setting. In the graph, the $\mathrm{x}$-axis represents the weight value, and the y-axis represents the amount of energy consumed. Because the difference in control packet numbers is very small and the energy consumed by the sink node to send data packets is reduced, the total energy consumed is the least. Elements to be considered when determining the most efficient weight value in terms of energy consumption include the number of control packets, average TPL, and total energy consumption. To put together, from Figures 7 and 8 in this paper, the difference in control packet numbers is very small except for when the weight is 0.5 . Therefore, data packets were sent with the most appropriate TPL on average, and a weight of 0.125 , which corresponds to the least energy consumption, was judged to be suitable. 


\subsection{Performance Evaluation of Previous TPC Algorithms}

Figure 9 shows the total energy consumption when each TPC algorithm is executed under the same body movement scenario. The X-axis of the graph represents TPC algorithm and the $y$-axis of the graph refers to the total energy consumption. Under the TPC algorithm proposed in this paper, the amount of energy consumed amount decreased in all elements, i.e., controlTX, dataRX, controlRX, and dataTX, which constitute the total energy consumption. By switching to a more appropriate TPL within a faster level with the proposed TPC algorithm, energy spent in sending/receiving data packets was saved, and by reducing the number of control packets in the algorithm, energy spent in sending/receiving control packets was saved. Resultantly, the total energy consumption was $523.48 \mathrm{~mJ}$, which indicates an energy efficiency improvement of $119 \%-145 \%$.

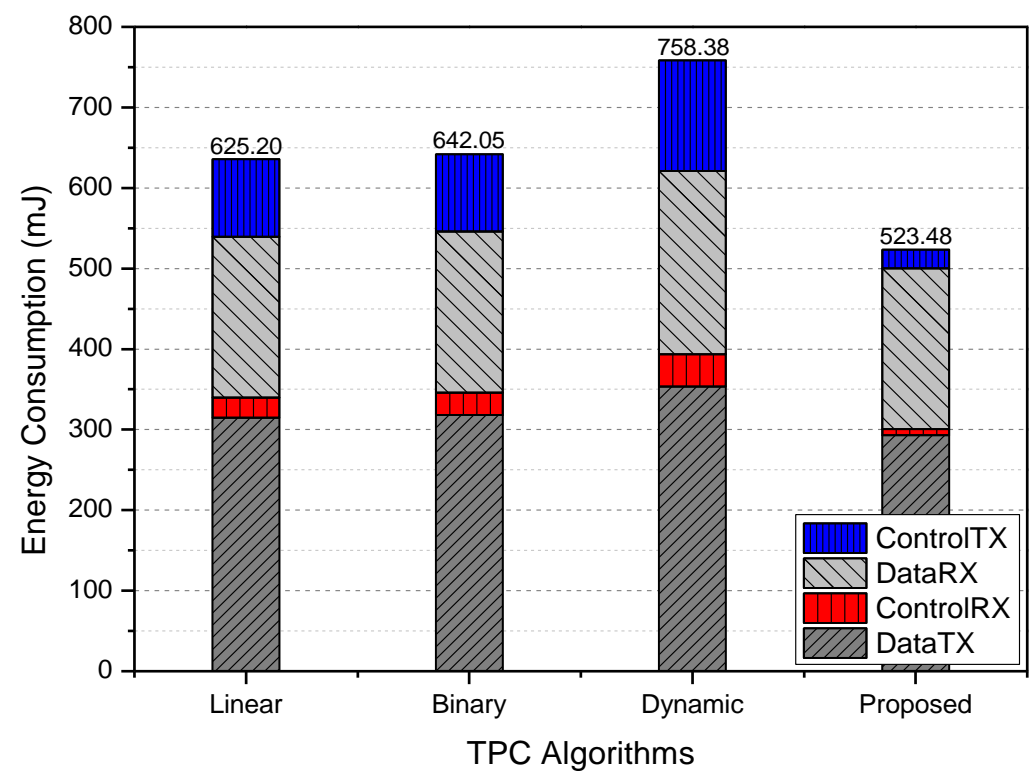

Figure 9. Total Energy Consumption According to TPC Algorithm

Next, the number of additionally lost packets and the number of control packets were analyzed comparatively. Figure 11 shows the numbers of lost packets and control packets in the representative TPC algorithms - linear, binary, and dynamic algorithms - and the algorithm proposed in this paper. The x-axis, y-axis on the left side, and y-axis on the right side of the graph represent the TPG algorithm, number of lost packets, and number of control packets, respectively. When a total of 720 packets are sent, the number of lost data packets in the four algorithms is $12,13,22$, and 10 , respectively, with the number of lost data packets being the largest with the dynamic algorithm and the least with the proposed algorithm. This shows that transmitting blindly on a low level does not reduce energy consumed in sending/receiving data packets. Besides, the proposed algorithm used 28 control packets, considerably fewer than the number of control packets used by the other TPC algorithms. Therefore, energy consumed for sending/receiving control packets is the least in the proposed algorithm. This effect is obtained by changing the standard for judging whether it goes beyond the target RSSI margin from the current RSSI to the average RSSI, which is determined using a moving average. 


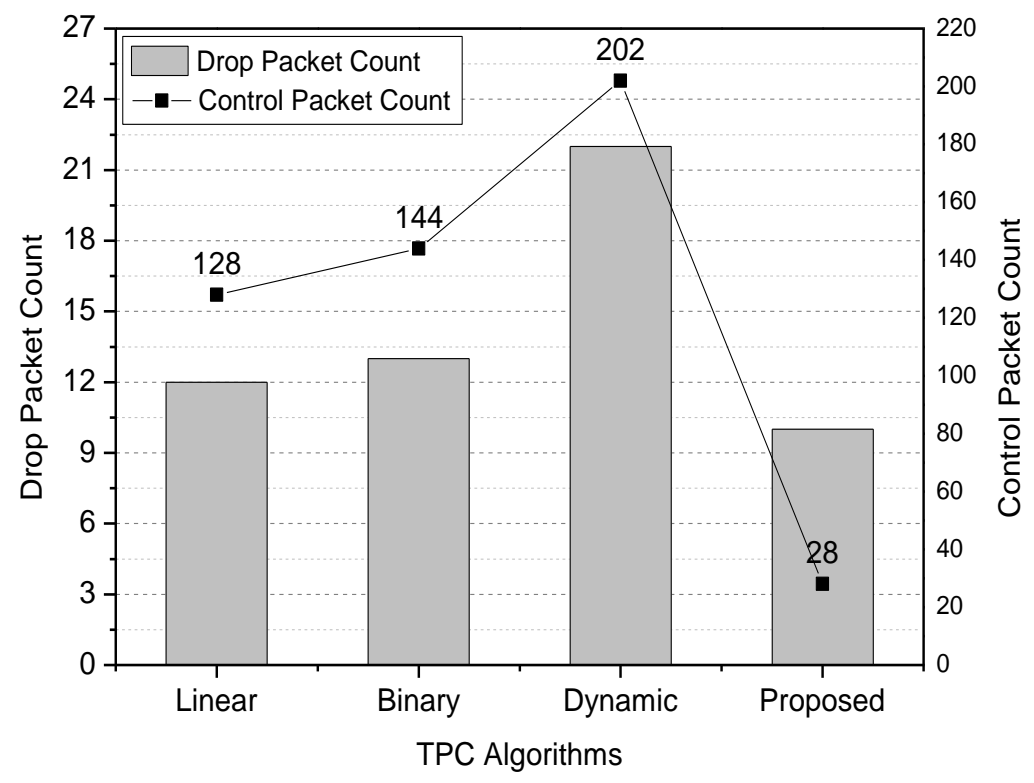

\section{Figure 10. Numbers of Control Packets and Drop Packets According to Algorithms}

\section{Conclusion}

In this paper, we proposed a measure to heighten the energy efficiency of nodes using TPC technology in a WSS. Changing TPL in a diversely changing channel environment may positively influence energy consumption. However, the biggest problem with existing TPC algorithms is that they send too many control packets. Moreover, they send control packets to an inappropriate TPL not suitable for the current channel environment and fail to find an appropriate TPL in a fast and precise manner. Accordingly, this paper proposed a new TPC algorithm that may minimize the energy consumed, number of control packets, and number of drop packets, thereby overcoming such a problem.

The proposed TPC algorithm determines a TPL appropriate for the channel environment within a faster level than existing TPC algorithms, thus reducing the number of unnecessary TPL changes and eliminating the phenomenon of oscillation failing to find an appropriate TPL. In the experiment described herein, the moving average technique was used. Here, the moving average weight corresponding to transmission of data packets to the most appropriate TPL with the least total energy consumption was 0.125 . In addition, the proposed TPC algorithm was able to reduce the total energy consumption by a maximum of $145 \%$ and a minimum of $119 \%$ compared with existing TPC algorithms. The proposed algorithm reduced control energy by more than 4.5 times on average compared with existing algorithms.

\section{Acknowledgment}

This work was supported by Kyonggi University Research Grant 2014.

\section{References}

[1] R. Jordan and C. A. Abdallah, "Wireless communications and networking: an overview", Report, Elect. and Comp. Eng. Dept., Univ., (2002); New Mexico.

[2] IEEE 802.15 WPAN WG homepage, http://www.ieee802.org/15.

[3] TG6 Jacksonville Plenary Meeting Minutes, IEEE 802.15-15-08-0393-01-0006

[4] I. F. Akyildiz, W. Su and E. Cayirci, "A survey on sensor networks", IEEE Communications Magazine, vol. 40 , no. 8, (2002).

[5] W. Ye, J. Heidemann and D. Estrin, "An energy-efficient MAC protocol for wireless sensor networks", INFOCOM, (2002). 
[6] H. Cotuk, K. Bicakci, B. Tavli and E. Uzun, "The Impact of Transmission Power Control Strategies on Lifetime of Wireless Sensor Networks", Computers IEEE Transactions on, vol. 63, no. 11, (2013).

[7] D. Knuth, "The Art of Computer Programming", Volume 3: Sorting and Searching 3rd ed., Addison-Wesley, (1998).

[8] T. H. Cormen and C. E. Leiserson, "Introduction to Algorithms 3rd ed", The MIT Press, (2009).

[9] M. Quwaider, J. Rao and S. Biswas, "Body-Posture-Based Dynamic Link Power Control in Wearable Sensor Networks", IEEE Communications Magazine, vol. 48, (2010).

[10] Crossbow-Technology, http://bullseye.xbow.com:81/index.as.

[11] CC1000 Data Sheet, http://www.ti.com/lit/ug/swru058/swru058.pdf.

[12] W.S Lee, M. Choi and N. Kim, "Different Characteristics of Radio Modules in Wireless Body Area Networks", Lecture Notes in Computer Science, vol. 7513, (2012).

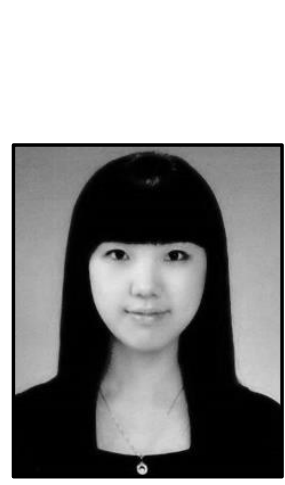

\section{Authors}

JinaHong received the B.S. degree in Computer Science from the Kyonggi University, Korea, in 2013. She is currently M.S. candidate in Computer Science from Kyonggi University. Her research interests include wireless systems, sensor networks.

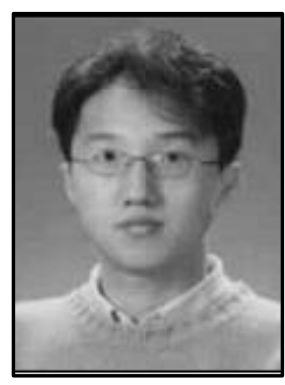

Namgi Kim received the B.S. degree in Computer Science from Sogang University, Korea, in 1997, and the M.S. degree and the Ph.D. degree in Computer Science from KAIST in 2000 and 2005, respectively. From 2005 to 2007, he was a research member of the Samsung Electronics. Since 2007, he has been a faculty of the Kyonggi University. His research interests include sensor system, wireless system, and mobile communication. 
International Journal of Control and Automation Vol. 8, No. 1 (2015) 